\title{
De novo transcriptomic analysis of Chlorella sorokiniana reveals differential genes expression in photosynthetic carbon fixation and lipid production
}

\author{
Lin Li, Guoqiang Zhang and Qinhong Wang ${ }^{*}$
}

\begin{abstract}
Background: Microalgae, which can absorb carbon dioxide and then transform it into lipid, are promising candidates to produce renewable energy, especially biodiesel. The paucity of genomic information, however, limits the development of genome-based genetic modification to improve lipid production in many microalgae. Here, we describe the de novo sequencing, transcriptome assembly, annotation and differential expression analysis for Chlorella sorokiniana cultivated in different conditions to reveal the change of genes expression associated with lipid accumulation and photosynthetic carbon fixation.

Results: Six cultivation conditions were selected to cultivate C. sorokiniana. Lipid content of C. sorokiniana under nitrogen-limited condition was 2.96 times than that under nitrogen-replete condition. When cultivated in light with nitrogen-limited supply, C. sorokiniana can use carbon dioxide to accumulate lipid. Then, transcriptome of C. sorokiniana was sequenced using Illumina paired-end sequencing technology, and 244,291,069 raw reads with length of $100 \mathrm{bp}$ were produced. After preprocessed, these reads were de novo assembled into 63,811 contigs among which 23,528 contigs were found homologous sequences in public databases through Blastx. Gene expression abundance under six conditions were quantified by calculating FPKM value. Ultimately, we found 385 genes at least 2-fold up-regulated while 71 genes at least 2-fold down-regulated in nitrogen-limited condition. Also, 204 genes were at least 2-fold up-regulated in light while 638 genes at least 2-fold down-regulated. Finally, 16 genes were selected to conduct RT-qPCR and 15 genes showed the similar results as those identified by transcriptomic analysis in term of differential expression.
\end{abstract}

Conclusions: De novo transcriptomic analyses have generated enormous information over C. sorokiniana, revealing a broad overview of genomic information related to lipid accumulation and photosynthetic carbon fixation. The genes with expression change under different conditions are highly likely the potential targets for genetic modification to improve lipid production and $\mathrm{CO}_{2}$ fixation efficiency in oleaginous microalgae.

Keywords: Chlorella sorokiniana, Transcriptome, Lipid accumulation, Photosynthetic carbon fixation, RNA-seq

\footnotetext{
* Correspondence: wang_qh@tib.cas.cn

Key Laboratory of Systems Microbial Biotechnology, Tianjin Institute of

Industrial Biotechnology, Chinese Academy of Sciences, 32 XiQiDao, Tianjin

Airport Economic Area, Tianjin 300308, People's Republic of China
} 


\section{Background}

The demand of energy is increasing as the world population and global economy continue to grow. Microalgae-based biodiesel, which can realize carbonneutral by photosynthetic carbon fixation via the microalgae's growth [1], is a renewable and sustainable energy source. Chlorella, one of eukaryotic, unicellular and photosynthetic microorganism, widely distributes in freshwater environment and is capable of accumulating excess lipid in nitrogen-limited condition. Moreover, Chlorella were used as a model system for investigating photosynthetic carbon fixation [2, 3]. Due to its various and robust metabolic capacities, Chlorella has received increasingly attention as promising microalgae to produce biomass [4], biodiesel [5] as well as high additional-value products [6].

Currently, Chlorella is one of the best microalgae as oil feedstock for the production of biodiesel [7]. Particularly, in nitrogen-limited condition, Chlorella can alter the metabolic pathways to accumulate a high proportion of lipid which can be used for biodiesel production [8-10]. Although the metabolic transition has been identified in the lipid accumulation process [11], many global changes remain poorly understood, such as genomic information, differential genes expression. As a consequence, the lipid production from naturally occurring Chlorella strains is much lower than the theoretical maximum [12], making the cost of biodiesel prohibitively high [13]. One primary cause is the limited understanding of the metabolic pathways in microalgae regulating the lipid metabolism in general and lipid biosynthesis and accumulation in particular [14]. Another cause is the lack of genomic information of some oleaginous but non-model microalgae, which largely hampers the investigation of the transcribed genes and genetic modification in these microalgae to accumulate lipid and other valuable products [15-17].

Transcriptome sequencing could be an efficient and relatively economical method to obtain the functional genomic information without the genomic information $[17,18]$, providing an initial, broad view of lipid accumulation in nitrogen-limited condition [15] and photosynthetic carbon fixation. A growing number of transcriptomes of oleaginous microalgae were de novo sequenced, assembled and annotated to investigate the regulatory mechanism of lipid accumulation [15-18].

In our previous work, we have already identified the metabolic pathways related to lipid accumulation in $C$. sorokiniana based on two transcriptome datasets [19]. In this present study, we sequenced another four transcriptome datasets and analyzed all six transcriptome datasets together to elucidate differential gene expression involved in the lipid accumulation and photosynthetic carbon fixation. In our experiments, the quantity of lipid accumulated under nitrogen-limited condition can be 2.96 times than that under nitrogen-replete condition, making C. sorokiniana a promising microalgae strain to produce biodiesel. Then all the six transcriptome datasets were de novo assembled, annotated together, and differential genes expression was analyzed as well. Finally, RT-qPCR was conducted for 16 genes involving in the lipid accumulation and photosynthetic carbon fixation. Our results provide an insight into the regulation of lipid metabolism and photosynthetic carbon fixation in $C$. sorokiniana at the transcriptomic level and may contribute to genetic modification in microalgae to improve lipid productivity and carbon fixation efficiency.

\section{Results and discussion}

Biomass and lipid content under different cultivation conditions

Six different cultivation conditions were selected to culture C. sorokiniana (Table 1), and the growth and lipid content profiles under these conditions were shown in Fig. 1. With $4 \%$ glucose as carbon source, the optical density of the culture at $680 \mathrm{~nm}\left(\mathrm{OD}_{680}\right)$ in nitrogenlimited condition $\left(0.2 \% \mathrm{KNO}_{3}\right.$ supply) was almost equal to that in nitrogen-replete condition $\left(0.8 \% \mathrm{KNO}_{3}\right.$ supply) before $48 \mathrm{~h}$. After $48 \mathrm{~h}$ the $\mathrm{OD}_{680}$ in nitrogenreplete condition began to become higher than that in nitrogen-limited condition (Fig. 1a). The higher $\mathrm{OD}_{680}$ resulted in the more glucose consumption (Fig. 1b) for cell growth, not for lipid production. The nitrogenlimited condition could induce $C$. sorokiniana to accumulate more lipid. After $48 \mathrm{~h}$, the fluorescence intensity of lipid dyed with nile red in nitrogen-limited condition was higher than that in nitrogen-replete condition, meaning that the cells in nitrogen-limited condition accumulated more lipid. At $84 \mathrm{~h}$, The fluorescence intensity in nitrogen-limited condition was as high as 2.96 times than that in nitrogen-replete condition $(340 \pm 19$ and $115 \pm 6$, respectively Fig. 1a).

When $C$. sorokiniana was cultivated in light, the fluorescence intensity increased steadily from $12.02 \pm 1.85$ at third day to $72.93 \pm 2.31$ at eighth day, increasing by 6.4 times and the $\mathrm{OD}_{680}$ increased continuously from $0.11 \pm$ 0.01 to $2.63 \pm 0.14$ over the whole cultivation period (Fig. 1c). These indicated that C. sorokiniana could absorb $\mathrm{CO}_{2}$ as carbon source to reproduce and also transform it into lipid accumulated in cells, which provided a promising strategy to alleviate global warming and energy crisis. When cultured heterotrophically in darkness with nitrogen-limited condition, C. sorokiniana accumulated lipid as well and the fluorescence intensity increased by 28.89 times at $84 \mathrm{~h}(324.72 \pm 32.09$, Fig. $1 \mathrm{~d})$. Compared with photoautotrophy, heterotrophy could 
Table 1 The general information for each sample

\begin{tabular}{|c|c|c|c|c|c|c|}
\hline & sample A & sample $B^{a}$ & sample C & sample $D^{a}$ & sample E & sample F \\
\hline $\mathrm{KNO}_{3}$ & $0.2 \%$ & $0.2 \%$ & $0.8 \%$ & $0.8 \%$ & $0.2 \%$ & $0.033 \%$ \\
\hline Carbon source & $4 \%$ glucose & $4 \%$ glucose & $4 \%$ glucose & $4 \%$ glucose & $4 \%$ glucose & $4 \%\left(\mathrm{CO}_{2} /\right.$ air, v/v) \\
\hline Temperature & $37^{\circ} \mathrm{C}$ & $37^{\circ} \mathrm{C}$ & $37^{\circ} \mathrm{C}$ & $37^{\circ} \mathrm{C}$ & $37^{\circ} \mathrm{C}$ & $\mathrm{RT}^{\mathrm{b}}$ \\
\hline Light & $-^{c}$ & - & - & - & dark & $100 \sim 120$ umol-photon $\cdot \mathrm{m}^{-2} \cdot \mathrm{s}^{-1}$ \\
\hline Fermentation time & $48 \mathrm{~h}$ & $84 \mathrm{~h}$ & $48 \mathrm{~h}$ & $84 \mathrm{~h}$ & $84 \mathrm{~h}$ & $8 d$ \\
\hline $\mathrm{OD}_{680}$ & $20.03 \pm 1.42$ & $24.83 \pm 1.45$ & $20.26 \pm 0.15$ & $26.10 \pm 0.61$ & $22.91 \pm 1.79$ & $2.63 \pm 0.09$ \\
\hline Fluorescence intensity $^{d}$ & $106.36 \pm 5.32$ & $340.42 \pm 19.13$ & $17.46 \pm 2.33$ & $115.31 \pm 7.60$ & $324.723 \pm 32.09$ & $72.93 \pm 2.31$ \\
\hline Shaking speed & 220 rpm & 220 rpm & 220 rpm & 220 rpm & 220 rpm & 0 rpm \\
\hline SRA accession number & SRX352462 & SRX354137 & SRX354139 & SRX354141 & SRX354143 & SRX354142 \\
\hline
\end{tabular}

${ }^{a}$ the transcriptome datasets of these samples were sequenced before

broom temperature $\left(25 \pm 2{ }^{\circ} \mathrm{C}\right)$

cdo not take the influence of light into account

${ }^{d}$ the fluorescence intensity of lipid dyed with nile red

make $C$. sorokiniana yield more biomass and achieve higher lipid productivity (Fig. 1c, d).

\section{Sequencing and de novo assembly}

After Illumina Hiseq2000 paired-end sequencing, over 244,291,069 raw reads were generated and are available at the NCBI SRA database (Table 1). All the raw reads were subjected to trimming based on base quality score and read length, and 229,288,757 clean reads were generated (Additional files 1 and 2), which were de novo assembled into 72,902 contigs with N50 of 2,502 bp. After clustered, 63,811 non-redundant contigs, ranging from $200 \mathrm{bp}$ to
$15,932 \mathrm{bp}$, were generated with an average length of 1,022 bp (Fig. 2a, Additional file 3), which was used for the following analysis. The Transcriptome Short Assembly project has been deposited at DDBJ/EMBL/GenBank under the accession GAPD00000000. The version described in this paper is the first version GAPD01000000.

\section{Annotation of contigs}

After compared against the NCBI's $\mathrm{Nr}$ database using Blastx, 23,496 contigs (36.8\% of total contigs) were found having homologous sequence in $\mathrm{Nr}$ database (Fig. 3, Additional file 4). Due to the lack of genome
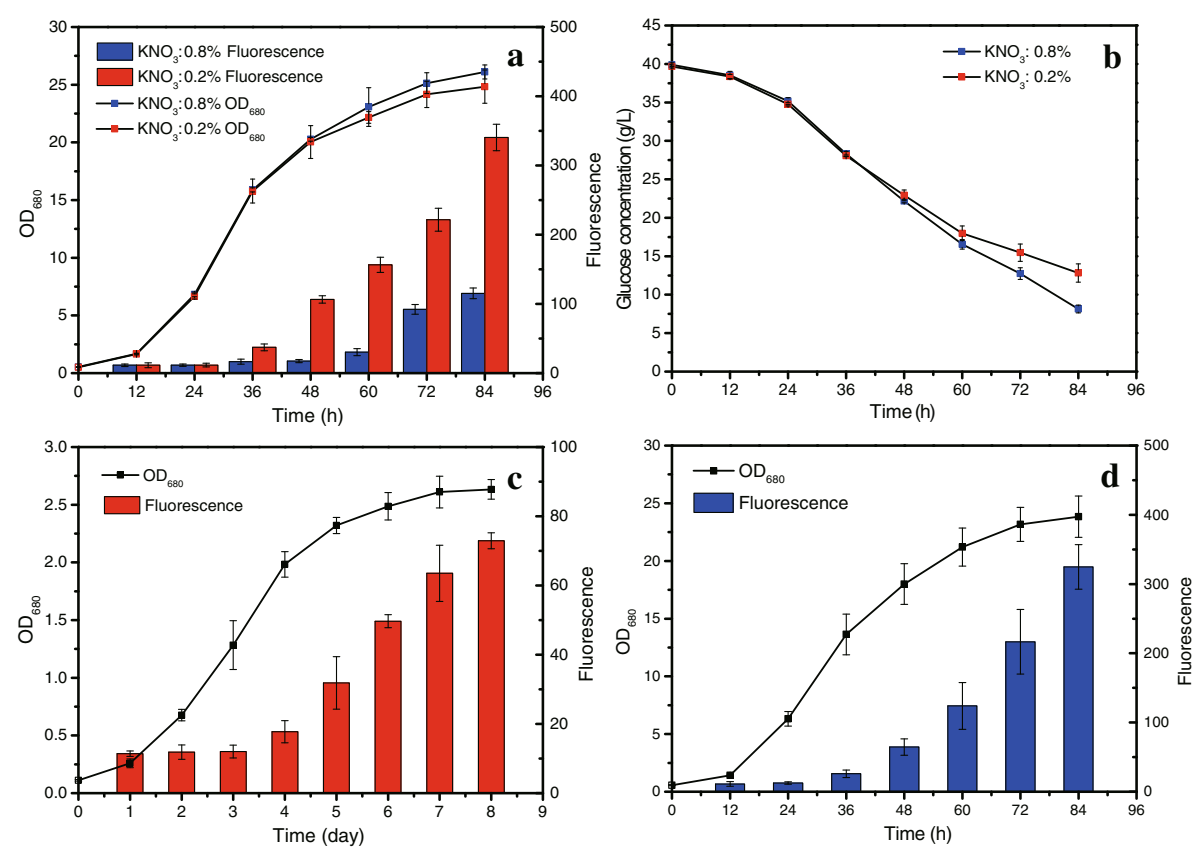

Fig. 1 Growth and lipid content of C. sorokiniana under different conditions. a: Growth and lipid content of C. sorokiniana under nitrogen-limited and nitrogen-replete conditions. b: The consumption of glucose under nitrogen-limited and nitrogen-replete conditions; c: Growth and lipid content of C. sorokiniana in light; $\mathbf{d}$ : Growth and lipid content of C. sorokiniana in dark 

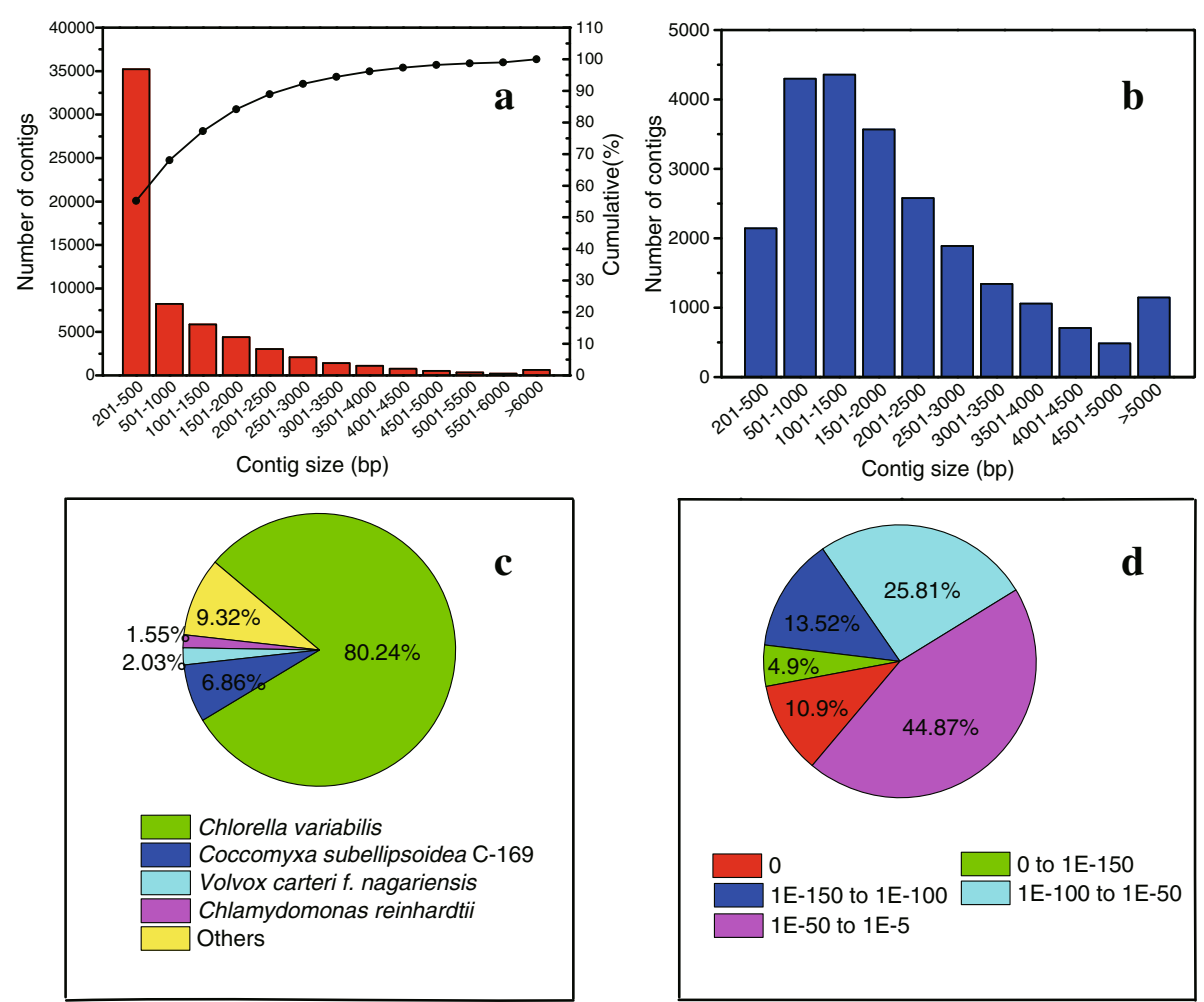

Fig. 2 Characteristics of homology search of assembly against the $\mathrm{Nr}$ database. a: The length distribution of contigs assembled. b: The length distribution of contigs with match (with a cut-off E-value of 1.0E-5) in Nr database. c: Species distribution of the total homologous sequences with E-value $\leq 1.0 \mathrm{E}-5$. d: E-value distribution of Blastx hits for each contigs with E-value $\leq 1.0 \mathrm{E}-5$. We used the first hit of each search results for analysis

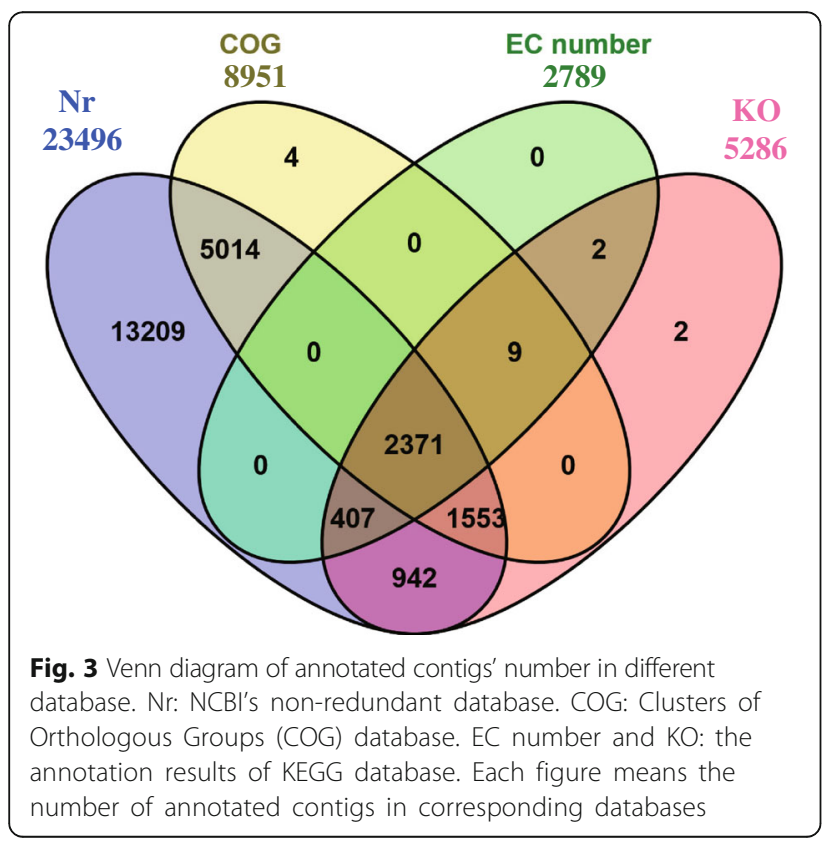

information, a large proportion of the contigs (40298, $63.2 \%)$ could not be matched to homologous sequence in any database, among which 10,471 potential coding regions were predicted using Transdecoder (Additional file 5). These predicted coding regions seem to be new genes, and their functions should be further confirmed. $\mathrm{EC}$ number and $\mathrm{KO}$ identifier were also assigned from the annotation results of KEGG, and 2,789 contigs were assigned with EC number (Fig. 3, Additional file 4). There were 2,371 contigs which were all matched with homologous sequences in all the databases used (Fig. 3). Particularly, the length of most contigs with homologous sequence in $\mathrm{Nr}$ database were between 500 and $2500 \mathrm{bp}$ (14801, $62.79 \%)$ and the match efficiency decreased as the length of contigs increased (Fig. 2b), indicating that most genes of $C$. sorokiniana were in the range of $500 \mathrm{bp}$ and 2500 bp. Moreover, the homologous sequences matched in $\mathrm{Nr}$ came from closely related green microalgae species, including $C$. variabilis (80.24 \% of all annotated contigs), Coccomyxa subellipsoidea C-169 (6.86 \%) and Volvox carteri f. nagariensis (2.03 \%) (Fig. 2c), based on which we selected Chlorella $s p$. NC64A as the candidate for predicting transcription factors. Similar results were also reported in the transcriptomic analysis of Dunaliella tertiolecta [16] and 
Chlamydomonas moewusii [20]. The E-value distribution of the top match in $\mathrm{Nr}$ database showed that $55.13 \%$ of the matched sequences had E-value $\leq 1.0 \mathrm{E}-50$, and $44.87 \%$ ranged from $1.0 \mathrm{E}-5$ to $1.0 \mathrm{E}-50$ (Fig. $2 \mathrm{~d}$ ). Similar results were also reported in the de novo transcriptomic analysis of Ambystoma mexicanum [21].

\section{Function classification and Transcription factor analysis}

8,951 contigs were assigned with at least 1 COG category and 13 contigs had no homologous sequence in $\mathrm{Nr}$ database but matched homologous sequence in COG database (Fig. 3). Among the 24 COG categories, the cluster for "general function prediction only" accounted for the largest group (2380, 19.22\%), followed by "Replication, recombination and repair" (1251, $10.1 \%)$ and "Transcription" (1000, $8.07 \%)$. In addition, the following categories contained the minimum number of contigs: "Nuclear structure" (1), "Cell motility" (11, $0.0009 \%)$, "RNA processing and modification" (77, $0.006 \%)$. We even did not find any contig assigned to the category "Extracellular structures" (Fig. 4, Additional file 4).

Transcription factors, most of which were protein, also play significant roles in responding to environment stress by regulating gene expression which were classified into different families such as SBP, C3H and bHLH. After searching against the transcription factors database of Chlorella sp. NC64A [22], 203 contigs were found having homologous sequences and assigned into 12 families (Table 2, Additional file 4). The most abundant transcription factor family was SBP family related to flower development in plant [23]. $\mathrm{C} 3 \mathrm{H}$ family was the next abundant transcription factor family, which played an important role during Arabidopsis embryogenesis and functioned primarily in the apical domain of the embryo [24] (Table 2). In terms of the specific function of these transcription factor families in C. sorokiniana, further studies should be further conducted. Moreover, the
Table 2 Transcription factor families identified in C. sorokiniana

\begin{tabular}{lll}
\hline Transcription factor family & Number of contigs & Number of TF ID \\
\hline ARR-B & 1 & 1 \\
bHLH & 3 & 2 \\
C2H2 & 6 & 2 \\
C3H & 21 & 13 \\
CPP & 6 & 2 \\
E2F-DP & 4 & 2 \\
G2-like & 11 & 6 \\
HB & 11 & 2 \\
MYB & 4 & 3 \\
MYB-related & 139 & 6 \\
SBP & 84 & 20 \\
WRKY & 2 & 1 \\
\hline
\end{tabular}

${ }^{\mathrm{a}}$ Transcription factor ID. In the Transcription Factor Database (PInTFDB), each transcriptome factor family contains several items and each item assigned with a TF ID

differential expression of transcription factor were also investigated. 27 contigs assigned to 20 different transcription factors were found at least 2-fold up-regulated while only 4 contigs homologous with 4 different transcription factors at least 2-fold down-regulated in nitrogen-limited condition. When C. sorokiniana was cultivated in light, we found 23 contigs homologous with 12 transcription factors at least 2-fold up-regulated and 40 contigs annotated as 17 transcription factors at least 2-fold down-regulated (Table 3, Additional file 4).

Up to now, it have been proved that Dof-type transcription factor and bHLH family have the function of regulating lipid accumulation in plants [25-27]. In this study, two transcription factors (IGS.gm_27_00071 and IGS.gm_8_00085) in bHLH family were identified and found both up-regulated in nitrogen-limited condition, which further confirm the significance of bHLH family

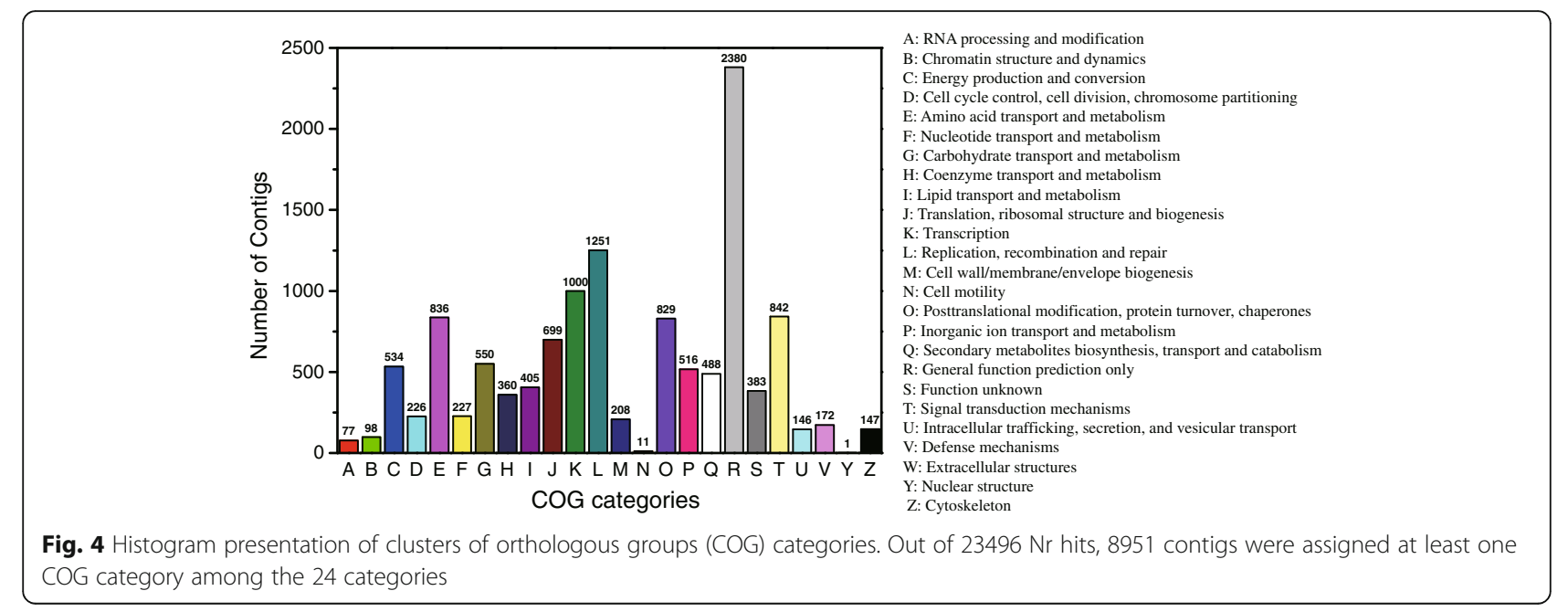


Table 3 The transcription factors with at least 2-fold expression change

\begin{tabular}{|c|c|c|c|}
\hline Transcription factor ID & Transcription factor family & Transcription factor ID & Transcription factor family \\
\hline \multicolumn{2}{|c|}{ up-regulated* in nitrogen-limited condition } & \multicolumn{2}{|l|}{ up-regulated in light } \\
\hline IGS.gm_27_00071 & bHLH & fgenesh3_pg.C_scaffold_21000059 & $\mathrm{C} 2 \mathrm{H} 2$ \\
\hline gw1.16.195.1 & $\mathrm{C} 3 \mathrm{H}$ & IGS.gm_27_00067 & $\mathrm{C} 3 \mathrm{H}$ \\
\hline IGS.gm_27_00067 & $\mathrm{C} 3 \mathrm{H}$ & estExt_fgenesh3_pg.C_130033 & $\mathrm{C} 3 \mathrm{H}$ \\
\hline IGS.gm_5_00352 & $\mathrm{C} 3 \mathrm{H}$ & IGS.gm_32_00066 & $\mathrm{C} 3 \mathrm{H}$ \\
\hline IGS.gm_14_00202 & $\mathrm{C} 3 \mathrm{H}$ & IGS.gm_15_00154 & G2-like \\
\hline IGS.gm_21_00108 & E2F-DP & IGS.gm_5_00099 & G2-like \\
\hline IGS.gm_16_00059 & G2-like & IGS.gm_5_00463 & G2-like \\
\hline IGS.gm_5_00099 & G2-like & estExt_fgenesh3_pg.C_240053 & MYB_related \\
\hline IGS.gm_15_00154 & G2-like & IGS.gm_28_00074 & SBP \\
\hline estExt_fgenesh3_pg.C_10352 & $\mathrm{HB}$ & IGS.gm_22_00154 & SBP \\
\hline fgenesh3_pg.C_scaffold_28000005 & MYB_related & IGS.gm_21_00140 & SBP \\
\hline gw1.9.308.1 & MYB_related & IGS.gm_27_00028 & SBP \\
\hline estExt_fgenesh3_pg.C_10164 & MYB_related & & \\
\hline IGS.gm_28_00074 & SBP & down-regulated in light & \\
\hline fgenesh3_pg.C_scaffold_6000074 & SBP & IGS.gm_5_00352 & $\mathrm{C} 3 \mathrm{H}$ \\
\hline IGS.gm_17_00084 & SBP & IGS.gm_3_00649 & $\mathrm{C} 3 \mathrm{H}$ \\
\hline IGS.gm_21_00140 & SBP & gw1.8.374.1 & $\mathrm{C} 3 \mathrm{H}$ \\
\hline IGS.gm_17_00054 & SBP & IGS.gm_26_00138 & CPP \\
\hline estExt_fgenesh3_pg.C_130036 & SBP & estExt_fgenesh3_pg.C_10352 & $\mathrm{HB}$ \\
\hline \multirow[t]{2}{*}{ fgenesh3_pg.C_scaffold_2000108 } & SBP & IGS.gm_4_00164 & $H B$ \\
\hline & & gw1.9.308.1 & MYB_related \\
\hline \multicolumn{2}{|c|}{ down-regulated ${ }^{\#}$ in nitrogen-limited condition } & estExt_Genewise1Plus.C_380072 & SBP \\
\hline estExt_fgenesh3_pg.C_130033 & $\mathrm{C} 3 \mathrm{H}$ & fgenesh3_pg.C_scaffold_9000137 & SBP \\
\hline estExt_Genewise1Plus.C_380072 & SBP & gw1.9.427.1 & SBP \\
\hline estExt_fgenesh3_pg.C_300018 & SBP & IGS.gm_13_00158 & $\mathrm{SBP}$ \\
\hline \multirow[t]{6}{*}{ estExt_fgenesh3_pg.C_130070 } & SBP & IGS.gm_17_00054 & SBP \\
\hline & & IGS.gm_17_00084 & SBP \\
\hline & & fgenesh3_pg.C_scaffold_25000062 & SBP \\
\hline & & estExt_fgenesh3_pg.C_130070 & SBP \\
\hline & & fgenesh3_pg.C_scaffold_6000074 & SBP \\
\hline & & estExt_fgenesh3_pg.C_130036 & SBP \\
\hline
\end{tabular}

${ }^{*}$ means at least 2-fold up-regulation

\#means at least 2-fold down-regulation

in the in lipid accumulation (Additional file 4). At the same time, others transcription factors assigned to other families were also found with at least 2-fold expression change in respond to nitrogen deprivation (Table 3), and most of these transcription factor families were also reported to be up-regulated in Chlamydomonas reinhardtii cultivated in $\mathrm{N}$-deprived condition especially the MYBrelated, SBP and $\mathrm{C} 3 \mathrm{H}$ family $[28,29]$. Thus, regulating these transcription factors would be a potential approach to increase the lipid accumulation [30, 31]. Moreover, many transcription factors related to photosynthetic carbon fixation were also found to be up-regulated or down- regulated in light (Table 3, Additional file 4). These results would be very useful for the improvement of photosynthetic carbon assimilation in microalgae as few transcription factors involving in photosynthetic carbon assimilation were investigated [32-34].

\section{Genes expression quantification}

The expression abundance of genes obtained from the annotation of assembled contigs were quantified using FPKM method [35]. When comparing genes expression abundance of sample A (nitrogen-limited condition, $48 \mathrm{~h}$ ) with that of sample C (nitrogen-replete condition, 
$48 \mathrm{~h}$ ), we found 533 genes were at least 2-fold upregulated and 219 genes were at least 2-fold downregulated in nitrogen-limited condition (Fig. 5, Additional file 6). Then comparing genes expression abundance between sample B (nitrogen-limited condition, $84 \mathrm{~h}$ ) and sample D (nitrogen-limited condition, $84 \mathrm{~h}$ ), 831 genes were found at least 2-fold up-regulated, while 171 genes down-regulated in sample B (Fig. 5, Additional file 6).

We also investigated the gene expression profiles for cultivation with $48 \mathrm{~h}$ and $84 \mathrm{~h}$, and found 385 genes at least 2-fold up-regulated in nitrogen-limited condition at both cultivation times (Fig. 5a), while 71 genes at least 2 -fold down-regulated (Fig. 5b). The expression change of most genes (1429 genes at $48 \mathrm{~h}, 1179$ genes at $84 \mathrm{~h}$, respectively) were less than 2 -fold, and 920 genes were found identical at both time (Fig. 5c). Interestingly, we found more genes with at least 2-fold up-regulation and less genes with at least 2-fold down-regulation at $84 \mathrm{~h}$ compared with the counterparts at $48 \mathrm{~h}$ (Fig. $5 \mathrm{a} \mathrm{b}$ ). The reason for this may be the concentration of nitrogen in the media declined with $C$. sorokiniana growing, which could induce more genes to increase its transcriptional level as a response to the lower concentration of nitrogen.

To investigate the differential expression of genes related to lipid accumulation, we mainly focused on the lipid-related metabolic pathways, including fatty acid biosynthesis and catabolism pathway, triacylglycerol biosynthesis pathway, and starch biosynthesis and catabolism pathway (Table 4). In these metabolic pathways, we found most genes up-regulated in nitrogen-limited condition at both $48 \mathrm{~h}$ and $84 \mathrm{~h}$ except those in starch metabolic pathway. On the contrary, the starch biosynthesis pathway was downregulated in nitrogen-limited condition, while the starch catabolic pathway was up-regulated, which was also reported in other microalgae [15], indicating microalgae preferred to synthesis lipid rather than starch in nitrogen-limited condition.

When investigating differential gene expression between light and dark condition, we found 842 genes with expression change more than 2-fold, comprising 204 genes up-regulated in light and 638 genes upregulated in dark. Similarly, the expression change of most genes (1342 genes) were less than 2-fold (Fig. 5d). In terms of photosynthetic carbon fixation, we particularly focused on the genes involving in Calvin cycle and found all genes except TPI (coding triosephosphate isomerase) up-regulated in light. The PGK (coding phosphoglycerate kinase), RBCL (coding ribulose-bisphosphate carboxylase large chain) and RPK (coding phosphoribulokinase) even found up-
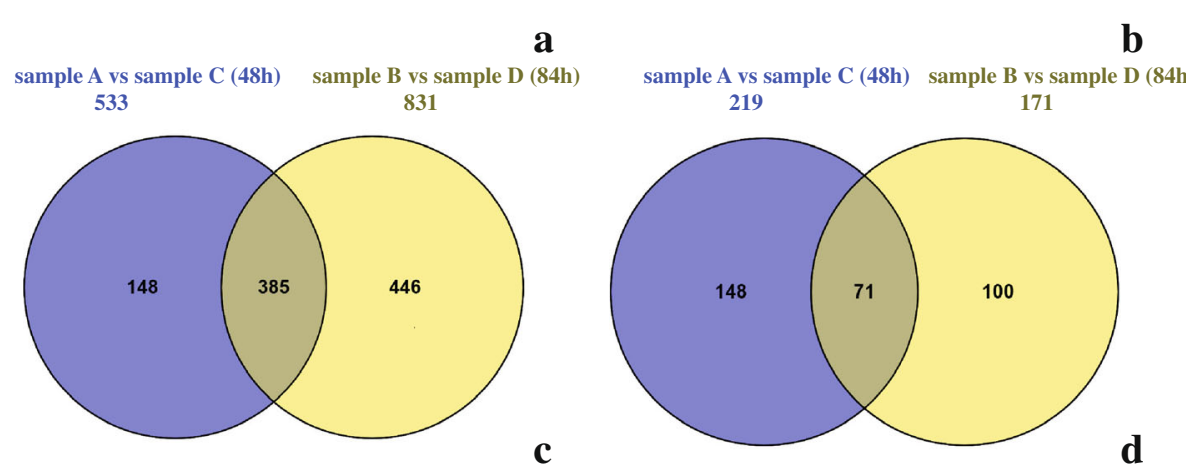

sample A vs sample C (48h) sample B vs sample D (84h)
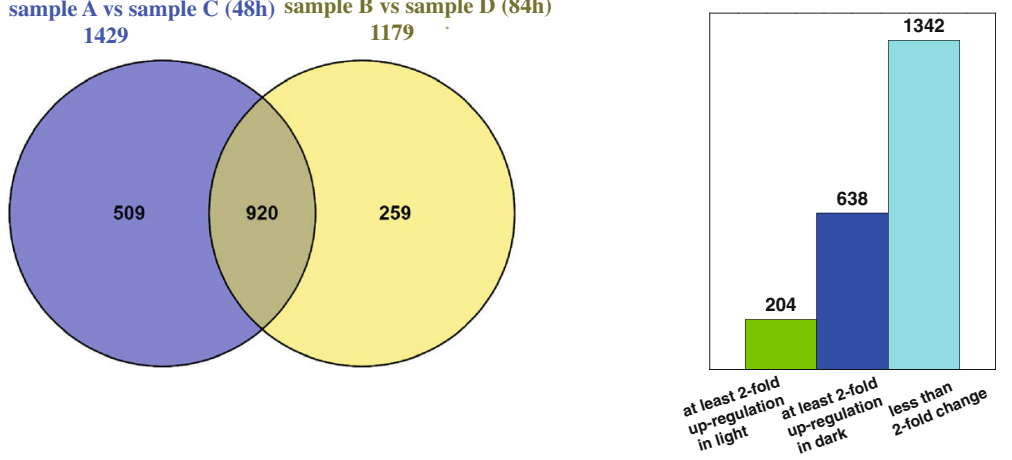

Fig. 5 Differential genes expression in C. sorokiniana of six cultivation conditions. a: At least 2-fold up-regulation in nitrogen-limited condition. b: At least 2-fold down-regulation in nitrogen-limited condition. c: No difference between nitrogen-limited and nitrogen-replete condition $(<2$-fold). d: Differential genes expression between light and dark condition 
Table 4 Differential gene expression in lipid accumulation related pathways

\begin{tabular}{|c|c|c|c|c|c|}
\hline Product & Gene name & EC number & Trinity ID & $\log _{2} \mathrm{FC}^{\mathrm{a}}(48 \mathrm{~h})$ & $\log _{2} \mathrm{FC}(84 \mathrm{~h})$ \\
\hline \multicolumn{6}{|l|}{ Fatty acid biosynthyesis pathway } \\
\hline acetyl-CoA carboxylase & ACC & EC:6.4.1.2 & comp6756_c0_seq1 & -1.71 & -0.63 \\
\hline biotin carboxylase & $B C$ & EC:6.3.4.14 & comp12323_c26_seq1 & 0.82 & 2.55 \\
\hline malonyl-CoA ACP transacylase & MAT & EC:2.3.1.39 & comp11367_c0_seq2 & -1.27 & -0.74 \\
\hline KAS Beta-ketoacyl-ACP synthase & KASIII & EC:2.3.1.179 & comp11672_c1_seq3 & 0.48 & 0.72 \\
\hline KAS Beta-ketoacyl-ACP synthase & KASIII & EC:2.3.1.180 & comp10141_c0_seq2 & 0.27 & 0.35 \\
\hline Beta-ketoacyl-ACP reductase & KAR & EC:1.1.1.100 & comp10720_c0_seq1 & 1.23 & 0.74 \\
\hline beta-hydroxyacyl-ACP dehydrase & HAD & EC:4.2.1.59 & comp12130_c0_seq1 & 0.31 & 1.43 \\
\hline Enoyl-ACP reductase & EAR & EC:1.3.1.10 & comp5575_c1_seq1 & -2.23 & 0.10 \\
\hline acyl-ACP desaturase & AAD & EC:1.14.19.2 & comp12618_c0_seq1 & 0.27 & 0.45 \\
\hline Oleoyl-ACP thioesterase & OAT & EC:3.1.2.14 & comp10287_c1_seq1 & 0.54 & 0.53 \\
\hline \multicolumn{6}{|l|}{ Triacylglycerol biosynthesis pathway } \\
\hline glycerol kinase & GK & EC:2.7.1.30 & comp17390_c0_seq1 & 1.21 & 1.12 \\
\hline glycerol-3-phosphate O-acyltransferase & GPAT & EC:2.3.1.15 & comp8649_c0_seq4 & 0.14 & 2.41 \\
\hline 1-acyl-sn-glycerol-3-phosphate acyltransferase & AGPAT & EC:2.3.1.51 & comp8576_c1_seq1 & 1.05 & 0.99 \\
\hline phosphatidate phosphatase & PP & EC:3.1.3.4 & comp10073_c0_seq1 & -0.75 & -3.36 \\
\hline diacylglycerol O-acyltransferase & DGAT & EC:2.3.1.20 & comp8627_c0_seq2 & 0.08 & 0.15 \\
\hline \multicolumn{6}{|l|}{ Fatty acid catabolism pathway } \\
\hline acyl-CoA synthetase & ACSL & EC:6.2.1.3 & comp11861_c1_seq8 & -0.57 & -0.29 \\
\hline acyl-CoA oxidase & $\mathrm{AOx}$ & EC:1.3.3.6 & comp8247_c0_seq2 & 2.17 & 1.24 \\
\hline acyl-CoA dehydrogenase & $\mathrm{ACDH}$ & EC:1.3.8.7 & comp12261_c15_seq6 & 0.62 & 1.22 \\
\hline enoyl-CoA hydratase & $\mathrm{ECH}$ & EC:4.2.1.17 & comp8664_c0_seq1 & 0.46 & 0.27 \\
\hline 3-hydroxyacyl-CoA dehydrogenase & $\mathrm{HADH}$ & $\mathrm{EC}: 1.1 .1 .35,1.1 .1 .211$ & comp13763_c0_seq1 & 1.64 & 1.97 \\
\hline acetyl-CoA C-acyltransferase & ACAT & EC:2.3.1.16, 2.3.1.9 & comp12241_c5_seq15 & 0.89 & 0.02 \\
\hline \multicolumn{6}{|c|}{ Starch biosynthesis and catabolism, and ethanol fermentation pathway } \\
\hline phosphoglucomutase & PGM & EC:5.4.2.2 & comp10999_c2_seq3 & -1.11 & -0.12 \\
\hline ADP-glucose pyrophosphorylase & AGPase & EC:2.7.7.27 & comp11635_c1_seq2 & 0.30 & 1.67 \\
\hline starch synthase & SS & EC:2.4.1.21 & comp13246_c0_seq1 & 0.04 & 0.30 \\
\hline 1,4-a-glucan branching enzyme & $\mathrm{BE}$ & EC:2.4.1.18 & comp7752_c1_seq1 & -1.47 & -1.66 \\
\hline hexokinase & HXK & EC:2.7.1.1 & comp5345_c0_seq1 & -0.20 & 1.49 \\
\hline$\beta$-amylase & $\beta$-AMY & EC:3.2.1.2 & comp13107_c0_seq1 & 1.49 & 1.36 \\
\hline a-amylase & a-AMY & EC:3.2.1.1 & comp11995_c1_seq7 & 2.22 & 1.10 \\
\hline oligo-1,6-glucosidase & $01,6 \mathrm{G}$ & EC:3.2.1.10 & comp11995_c1_seq11 & 2.20 & 1.49 \\
\hline starch phosphorylase & Spase & EC:2.4.1.1 & comp12085_c7_seq13 & 0.79 & 1.47 \\
\hline pyruvate decarboxylase & PDC & EC:4.1.1.1 & comp6820_c0_seq2 & -0.30 & 0.93 \\
\hline alcohol dehydrogenase & $\mathrm{ADH}$ & EC:1.1.1.1 & comp11611_c1_seq3 & 0.71 & 1.13 \\
\hline Pyruvate dehydrogenase complex & $\mathrm{PDHC}$ & EC:1.2.4.1, 2.3.1.12, 1.8.1.4 & comp12893_c0_seq1 & 1.48 & 1.90 \\
\hline
\end{tabular}

${ }^{a} \log 2 \mathrm{FC}=\log 2\left(\frac{F P K M n i t r o g e n-l i m i t e d}{\text { FPKMnitrogen-replete }}\right)$

regulated by $10.6,5.42$ and 4.66 times, respectively (Table 5). Unfortunately, there were still many genes with differential expression annotated as "hypothetical protein with unknown functions" in the annotation results (Additional file 6). Therefore, it will be necessary to investigate the potential functions of these genes.

\section{Real-time quantitative PCR analysis}

16 genes were selected to perform Real-time quantitative PCR (RT-qPCR). In the lipid metabolic pathways (Fig. 6a), 6 genes (biotin carboxylase, BC; 3-oxoacyl[acyl-carrier-protein] synthase II, KAS II; 3-oxoacyl[acyl-carrier-protein] synthase II, KAS III; Beta-ketoacyl- 
Table 5 Differential gene expression in Calvin cycle

\begin{tabular}{llll}
\hline Product & Gene name & EC number & Trinity ID \\
\hline ribulose-bisphosphate carboxylase large chain & RBCL & EC:4.1.1.39 & comp10529_c0_seq1 \\
fructose-bisphosphate aldolase, class I & ALDO & EC:4.1.2.13 & comp12064_c4_seq2 \\
sedoheptulose-1,7-bisphosphatase & SEBP & EC:3.1.3.37 & comp8533_c0_seq1 \\
transketolase & TRK & EC:2.2.1.1 & comp12612_c0_seq1 \\
ribose 5-phosphate isomerase A & RPIA & EC:5.3.1.6 & comp14985_c0_seq1 \\
phosphoribulokinase & RPK & EC:2.7.1.19 & comp13013_c0_seq1 \\
ribulose-phosphate 3-epimerase & RPE & EC:5.1.3.1 & comp12802_c0_seq1 \\
phosphoglycerate kinase & PGK & EC:2.7.2.3 & comp11827_c0_seq2 \\
glyceraldehyde-3-phosphate dehydrogenase & GAPDH & EC:1.2.1.13 & comp11943_c0_seq2 \\
triosephosphate isomerase & TPI & EC:5.3.1.1 & comp8453_c0_seq1 \\
fructose-1,6-bisphosphatase & FBPase & EC:3.1.3.11 & comp10769_c0_seq3
\end{tabular}

${ }^{a} \log 2 \mathrm{FC}=\log 2\left(\frac{F P K M \text { light }}{\text { FPKMdark }}\right)$
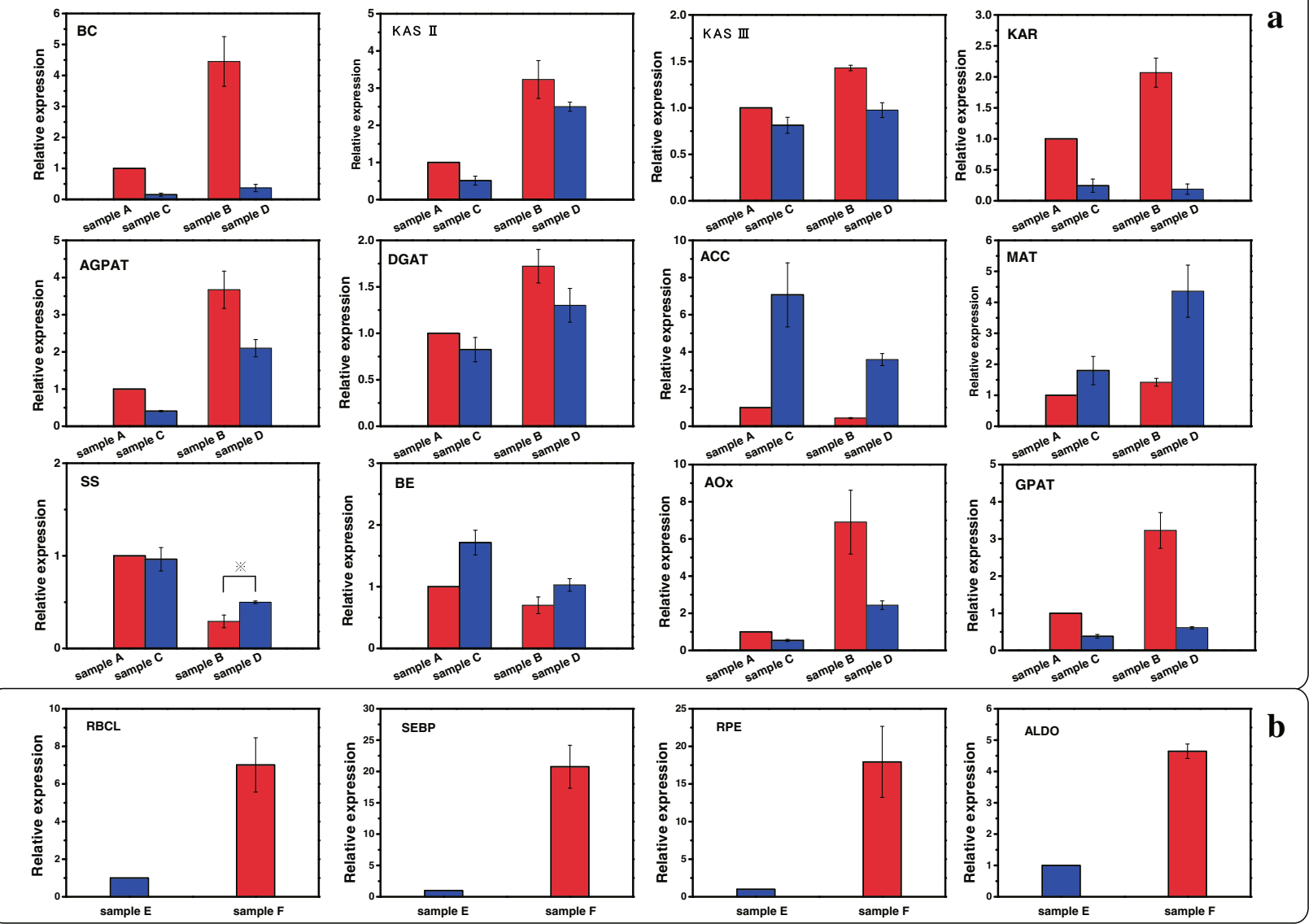

Fig. 6 The differentially expressed genes profiles detected by RT-qPCR. C. sorokiniana was cultivated under six different conditions. a: genes involving in lipid accumulation; b: genes involving in Calvin cycle. BC: biotin carboxylase; KAS II: 3-oxoacyl-[acyl-carrier-protein] synthase II; KAS III: 3-oxoacyl-[acylcarrier-protein] synthase III; KAR: Beta-ketoacyl-ACP reductase; AGPAT: 1-acyl-sn-glycerol-3-phosphate acyltransferase; DGAT: diacylglycerol O-acyltransferase; ACC: acetyl-CoA carboxylase; MAT: malonyltransferase; SS: starch synthase; BE: 1,4-a-glucan branching enzyme; AOx: acyl-CoA oxidase; GPAT: glycerol-3-phosphate O-acyltransferase; RBCL: ribulose-bisphosphate carboxylase large chain; SEBP: sedoheptulosebisphosphatase; RPE: ribulose-phosphate 3-epimerase; ALDO: fructose-bisphosphate aldolase. Standard error of mean for three technical replicates is represented by the error bars 
[acyl-carrier-protein] reductase, KAR; 1-acyl-sn-glycerol-3-phosphate acyltransferase, AGPAT; diacylglycerol O-acyltransferase, DGAT) showed up-regulation in nitrogen-limited condition, especially $B C$ and KAR. However, 2 genes (acetyl-CoA carboxylase, ACC; malonyltransferase, MAT) were found down-regulated in the nitrogen-limited condition. The down-regulation of ACC and up-regulation of BC under nitrogenlimited condition were also reported in Neochloris oleoabundans [15].

Moreover, we found the down-regulation of starch biosynthesis pathway (starch synthase, SS; 1,4- $\alpha$-glucan branching enzyme, BE) in nitrogen-limited condition, indicating that the starch biosynthesis pathway might be inhibited (Fig. 6a). This result was also reported in $N$. oleoabundans [15]. 4 genes involving in carbon fixation pathway (ribulose-bisphosphate carboxylase large chain, RBCL; sedoheptulose-bisphosphatase, SEBP; ribulosephosphate 3-epimerase, RPE; fructose-bisphosphate aldolase, ALDO) were found all up-regulated in light (Fig. 6b).

Among the 16 genes with RT-qPCR analysis, 15 genes showed the similar expression patterns as those identified by the transcriptomic analysis. Only the gene coding starch synthase showed inconsistent result in term of differential expression between the RT-qPCR analysis and transcriptomic analysis at $84 \mathrm{~h}$. Based on the result of RT-qPCR analysis, this gene was found downregulated in nitrogen-limited condition at $84 \mathrm{~h}$, while the transcriptomic analysis result showed it was upregulated in the corresponding condition.

\section{Conclusions}

This study not only provided transcriptome datasets of C. sorokiniana under six different conditions, but also new biological insights into the expression of genes associated with lipid accumulation and photosynthetic carbon fixation. Based on our study, it is clear that the application of this approach can contribute to the generation of interesting hypotheses for both fundamental and applied research. Moreover, the C. sorokiniana's transcriptome data could be a contribution for elucidating the physiology and evolution of the chlorophyceans.

\section{Methods}

\section{Strain and culture conditions for RNA-seq}

C. sorokiniana (UTEX 1602) was obtained from the Culture Collection of Alga at University of Texas (UTEX, Austin, TX, USA) and cultivated using modified Kuhl medium (Additional file 7). To induce the differential expression of genes involving in lipid accumulation and photosynthetic carbon fixation, six conditions were selected for transcriptome sequencing, including: $0.2 \%$ nitrate supply with cultivation of $48 \mathrm{~h}$ (nitrogen-limited, sample A); $0.2 \%$ nitrate supply with cultivation of $84 \mathrm{~h}$ (nitrogen-limited, sample B); $0.8 \%$ nitrate supply with cultivation of $48 \mathrm{~h}$ (nitrogen-replete, sample C); $0.8 \%$ nitrate supply with cultivation of $84 \mathrm{~h}$ (nitrogen-replete, sample D); $0.2 \%$ nitrate supply with cultivation in darkness of $84 \mathrm{~h}$ (heterotrophy with nitrogen-limited, sample E); $0.033 \%$ nitrate supply, white fluorescent light (100 $120 \mu \mathrm{mol}$ photons $\cdot \mathrm{m}^{-2} \cdot \mathrm{s}^{-1}$ ) and agitation by air containing $4 \%(\mathrm{v} / \mathrm{v}) \mathrm{CO}_{2}$ with cultivation of $8 \mathrm{~d}$ (photoautotrophy with nitrogen-limited, sample F) (Table 1). The transcriptomes of sample A, C, E and F were sequenced in this study and the datasets of sample B and D were sequenced before (SRX354137 and SRX354141, respectively) [19], which we analyze together with the purpose of getting the most comprehensive transcript pool using de novo assembly method.

In this study, we selected six experimental conditions to compare the expression level of genes and each experimental condition have one biological replicate $(n=$ 1). To keep the concordance of cultivation, the culture method of Sample A, C and E was the same as that of sample B and D [19], using $250 \mathrm{~mL}$ Erlenmeyer flask with $100 \mathrm{~mL}$ medium shaking at $220 \mathrm{rpm}$ at $37{ }^{\circ} \mathrm{C}$. Sample F was cultivated using $\Phi 1 \times 50 \mathrm{~mm}$ Cylindrical glass tube with $300 \mathrm{~mL}$ medium agitating with air at room temperature $\left(25 \pm 2{ }^{\circ} \mathrm{C}\right)$. After cultivation, cells were harvested by centrifugation (Eppendorf, Germany) at $4000 \mathrm{rpm}$, for $5 \mathrm{~min}$, at $4{ }^{\circ} \mathrm{C}$. The cell pellets were immediately frozen in liquid nitrogen and stored at $-80{ }^{\circ} \mathrm{C}$ until further analysis.

\section{RNA extraction, library construction and sequencing}

Total RNA of four samples (sample A, C, E and F) were extracted separately using General Total RNA Extraction Kit (QIAGEN, Germany) according to the manufacturer's instructions. After the elimination of the contaminant DNA, oligo ( $\mathrm{dT}$ ) beads were used to isolate mRNA from total RNA, followed by mRNA was cut randomly into short fragments. These fragmented RNA was reverse-transcribed to the first-strand cDNA with reverse transcriptase (Invitrogen, USA) that was then used as template to synthesis the second-strand of cDNA with DNA polymeraseland RNase H (Invitrogen). The resulting short cDNA fragments were purified using QiaQuik PCR Extraction Kit (QIAGEN) and resolved in an elution buffer for end reparation and addition of a single adenine base to 3'ends. Then the cDNA fragments were linked with sequencing adapters and separated in gels by electrophoresis. The fragments with a desirable size were cut from gels and eluted for PCR amplification. After qualified with Agilent 2100, each cDNA library was sequenced with Illumina Hiseq2000 platform (Illumina, USA). These RNA extraction and library construction processes were the same as those used for sample B and D. 


\section{Analysis of biomass and lipid content}

The biomass of $C$. sorokiniana was determined by measuring the $\mathrm{OD}_{680}$ using the microplate reader (Molecular Devices, USA). Lipid content was determined using the modified nile red staining method [36]. The culture was diluted with corresponding medium until the $\mathrm{OD}_{680}$ was between 0.1 and 0.3 . Then $1 \mathrm{~mL}$ of this algal suspension was stained with $3.33 \mu \mathrm{L}$ nile red solution $\left(7.8 \times 10^{-4}\right.$ $\mathrm{mol} \cdot \mathrm{L}^{-1}$ dissolved in acetone) and then excited at $486 \mathrm{~nm}$ before measuring the emission at $570 \mathrm{~nm}$ using the microplate reader. Glucose concentration was measured using HPLC method (Agilent Technologies, USA).

Preprocessing, de novo assembly and function annotation The 100 bp paired-end raw reads generated from Illumina Hiseq2000 were analyzed by FastQC tool (v0.10.1) [37] for quality assessment and preprocessed using Python scripts (Additional file 8), including: (a) remove low quality bases with Phred score $<20$, (b) remove ambiguous base 'N', (c) discard short reads with length < $25 \mathrm{bp}$. Followed by the high quality reads were de novo assembled using Trinity (v2.0.6) [38] with default parameters to construct contigs. Final clustering of contigs were conducted using the Cluster Database at High Identity with Tolerance (CD-HIT) EST suits [39] with minimum similarity cut-off of $90 \%$ to generate the nonredundant contigs used for the following analysis.

For the functional annotation, the non-redundant contigs were searched against with the NCBI's nonredundant $(\mathrm{Nr})$ database and Clusters of Orthologous Groups (COG) database [40, 41] using Blastx algorithm [42] with $E$-value $\leq 10^{-5}$ and $10^{-10}$, respectively, and other default parameters. Putative gene function, coding sequence and predicted proteins of corresponding contigs could be obtained by parsing the features of the best hit from each Blastx result. For the contigs that had no hit in any databases, the Transdecoder was used to predict potential coding sequences with default parameters. The Blastx results from COG database were used to identify the cluster of orthologous groups. To identify BRITE functional hierarchies [43], the non-redundant contigs were also submitted to the KEGG Automatic Annotation Server (KAAS) [44] with bi-directional best hit assignment method. KAAS could annotate each submitted sequence with KEGG orthology (KO), corresponding enzyme commission number (EC number) with the threshold of Blast bit scores $>60$. Putative transcription factors were also identified by searching Plant Transcription Factor Database (PlnTFDB) [45] using Blastx algorithm [42] with $E$-value Blas ${ }^{-10}$. Chlorella $s p$. NC64A [22] was selected as the candidate to search against in order to predict the transcription factors in $C$. sorokiniana.

\section{Gene expression quantification}

To determine the gene expression abundance, high quality reads from each condition were mapped to the nonredundant contigs to calculate the FPKM value [35] using the RSEM (v1.2.7) [46]. Due to the lack of biological replicates, we selected genes whose FPKM value was greater than 0 in all six conditions to study the differential expression and genes with the change of FPKM value greater than 2-fold in comparison of two different conditions were identified as differential expression.

\section{Real-time quantitative PCR}

In order to avoid the bias caused by the absence of biological replicates, we selected 16 genes, involving in lipid accumulation and carbon fixation, to perform the RTqPCR. The same conditions were used to cultivate $C$. sorokiniana for the RT-qPCR analysis. M-MLV reverse transcription kit (Promega, USA) was used to synthesis the cDNA according to the manufacturer's instruction. Gene specific primers (Additional file 9) for RT-qPCR were designed using Vecter NTI software. A $10 \mu \mathrm{L}$ reaction system was performed on the Eco real-time PCR system (Illumina, USA) with the absolute SYBR Green qPCR Kit Master Mix (Toyobo, Japan) according to the manufacturer's instruction. The cycle threshold value (CT) was determined and differential expression was calculated using the $2^{-\triangle \Delta C T}$ method [47] with $18 \mathrm{~S}$ gene of C. sorokiniana as the endogenous reference. Each sample was run in triplicate to confirm the reproducibility of the results.

\section{Additional files}

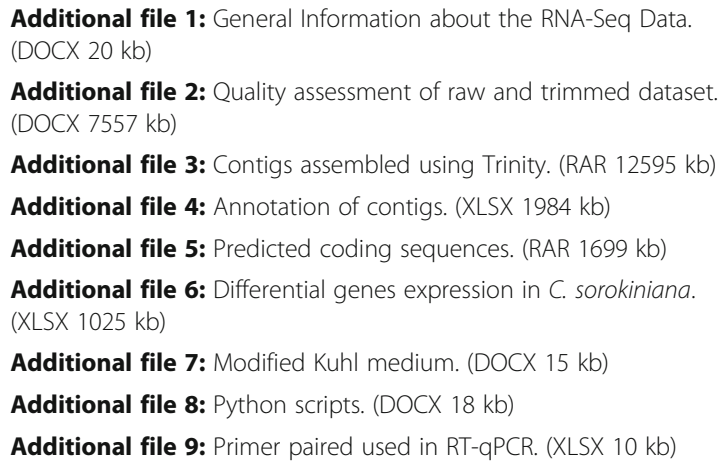

Acknowledgements

This work was supported by the National Basic Research Program of China (973 Program, 2011CB200900).

\section{Authors' contributions}

$\mathrm{LL}$ did the experiments, analyzed the transcriptome data and prepared the manuscript; GZ did the experiments; QW designed the experiments, discussed the results and revised the manuscript for publication. All authors read and approved the final manuscript. 


\section{Competing interests}

The authors declare that they have no competing interests.

\section{Received: 17 April 2015 Accepted: 14 September 2016} Published online: 26 September 2016

\section{References}

1. Schenk P, Thomas-Hall S, Stephens E, Marx U, Mussgnug J, Posten C, Kruse O, Hankamer B. Second Generation Biofuels: High-Efficiency Microalgae for Biodiesel Production. Bioenerg Res. 2008;1(1):20-43.

2. Bassham JA, Benson AA, Calvin M. The path of carbon in photosynthesis J Biol Chem. 1950;185:781-7.

3. Barker SA, Bassham JA, Calvin M, Quarck UC. Intermediates in the photosynthetic cycle. Biochim Biophys Acta. 1956;21(2):376-7.

4. Lee Y-K. Microalgal mass culture systems and methods: Their limitation and potential. J Appl Phycol. 2001;13(4):307-15.

5. $\mathrm{Xu} \mathrm{H}$, Miao X, Wu Q. High quality biodiesel production from a microalga Chlorella protothecoides by heterotrophic growth in fermenters. J Biotechnol. 2006;126(4):499-507.

6. Pulz O, Gross W. Valuable products from biotechnology of microalgae. Appl Microbiol Biotechnol. 2004;65(6):635-48

7. Gouveia L, Oliveira A. Microalgae as a raw material for biofuels production. J Ind Microbiol Biotechnol. 2009;36(2):269-74.

8. Miao X, Wu Q. Biodiesel production from heterotrophic microalgal oil. Bioresour Technol. 2006;97(6):841-6

9. Griffiths M, Harrison SL. Lipid productivity as a key characteristic for choosing algal species for biodiesel production. J Appl Phycol. 2009;21(5):493-507.

10. Chisti Y. Biodiesel from microalgae. Biotechnol Adv. 2007;25(3):294-306.

11. Xiong W, Liu L, Wu C, Yang C, Wu Q. 13C-tracer and gas chromatographymass spectrometry analyses reveal metabolic flux distribution in the oleaginous microalga Chlorella protothecoides. Plant Physiol. 2010;154(2):1001-11.

12. Hu Q, Sommerfeld M, Jarvis E, Ghirardi M, Posewitz M, Seibert M, Darzins A. Microalgal triacylglycerols as feedstocks for biofuel production: perspectives and advances. Plant J. 2008:54(4):621-39.

13. Acién FG, Fernández JM, Magán JJ, Molina E. Production cost of a real microalgae production plant and strategies to reduce it. Biotechnol Adv. 2012;30(6):1344-53.

14 Li Y, Han D, Sommerfeld M, Hu Q. Photosynthetic carbon partitioning and lipid production in the oleaginous microalga Pseudochlorococcum sp. (Chlorophyceae) under nitrogen-limited conditions. Bioresour Technol. 2011;102(1):123-9.

15 Rismani-Yazdi H, Haznedaroglu BZ, Hsin C, Peccia J: Transcriptomic analysis of the oleaginous microalga Neochloris oleoabundans reveals metabolic insights into triacylglyceride accumulation. Biotechnol Biofuels 2013

16 Rismani-Yazdi H, Haznedaroglu BZ, Bibby K, Peccia J. Transcriptome sequencing and annotation of the microalgae Dunaliella tertiolecta: pathway description and gene discovery for production of next-generation biofuels. BMC Genomics. 2011:12:148.

17 Zheng M, Tian J, Yang G, Zheng L, Chen G, Chen J, Wang B. Transcriptome sequencing, annotation and expression analysis of Nannochloropsis sp. at different growth phases. Gene. 2013;523(2):117-21.

18 Guarnieri MT, Nag A, Smolinski SL, Darzins A, Seibert M, Pienkos PT. Examination of triacylglycerol biosynthetic pathways via de novo transcriptomic and proteomic analyses in an unsequenced microalga. PLoS One. 2011;6(10):e25851.

19 Li L, Wang Q, Yang H, Wang W: De novo transcriptomic analysis of Chlorella sorokiniana: Pathway description and gene discovery for lipid production. Acta Microbiologica Sinica 2014, 54(9):1010-1021

20 Yang S, Guarnieri MT, Smolinski S, Ghirardi M, Pienkos PT: De novo transcriptomic analysis of hydrogen production in the green alga Chlamydomonas moewusii through RNA-Seq. Biotechnol biofuels $2013,6: 118-118$

21 Wu CH, Tsai MH, Ho CC, Chen CY, Lee HS. De novo transcriptome sequencing of axolotl blastema for identification of differentially expressed genes during limb regeneration. BMC Genomics. 2013;14:434-46.

22 Blanc G, Duncan G, Agarkova I, Borodovsky M, Gurnon J, Kuo A, Lindquist E, Lucas S, Pangilinan J, Polle J, et al. The Chlorella variabilis NC64A genome reveals adaptation to photosymbiosis, coevolution with viruses, and cryptic sex. Plant Cell. 2010;22(9):2943-55.
23 Yamasaki K, Kigawa T, Inoue M, Tateno M, Yamasaki T, Yabuki T, Aoki M, Sek E, Matsuda T, Nunokawa E, et al. A novel zinc-binding motif revealed by solution structures of DNA-binding domains of Arabidopsis SBP-family transcription factors. J Mol Biol. 2004;337(1):49-63.

24 Li Z, Thomas TL. PEl1, an Embryo-Specific Zinc Finger Protein Gene Required for Heart-Stage Embryo Formation in Arabidopsis. Plant Cell Online. 1998;10(3):383-98.

25 Wang H-W, Zhang B, Hao Y-J, Huang J, Tian A-G, Liao Y, Zhang J-S, Chen S-Y. The soybean Dof-type transcription factor genes, GmDof4 and GmDof11, enhance lipid content in the seeds of transgenic Arabidopsis plants. Plant J. 2007:52(4):716-29.

26 Kamisaka Y, Tomita N, Kimura K, Kainou K, Uemura H. DGA1 (diacylglycero acyltransferase gene) overexpression and leucine biosynthesis significantly increase lipid accumulation in the Deltasnf2 disruptant of Saccharomyces cerevisiae. Biochem J. 2007;408(1):61-8.

27 Riano-Pachon DM, Correa LG, Trejos-Espinosa R, Mueller-Roeber B. Green transcription factors: a chlamydomonas overview. Genetics. 2008;179(1):31-9.

28 Rachel M, Guangxi W, Rahul RD, Astrid V, Katrin G, Xiaobo L, Eric RM, Simone A, Adam JC, Bensheng L, et al. Changes in Transcript Abundance in Chlamydomonas reinhardtii following Nitrogen Deprivation Predict Diversion of Metabolism. Plant Physiol. 2010;154(4):1737-52.

29 Hexin L, Ge Q, Xizhen Q, Lina L, Chaoguang T, Yanhe M. Transcriptome analysis of Chlamydomonas reinhardtii during the processof lipid accumulation. Genomics. 2013;101(4):229-37.

30 Noemie MDC, Albert P, Bei W, Christopher QL. Enhancement of lipid production using biochemical, genetic and transcription factor engineering approaches. J Biotechnol. 2009;141(1-2):31-41.

31 Alejandro IS, Sergio RM, Alejandro RU, Jocelin IRA, Ignacio LH, Araceli HT. Over-expression of Dof-type transcription factor increases lipidproduction in Chlamydomonas reinhardtii. J Biotechnol. 2014;183:27-38.

32 Silvia P, Enrique F, Antonia H. The LysR-type transcription factor PacR is a global regulator of photosynthetic carbon assimilation in Anabaena. Environ Microbiol. 2015;17(9):3341-51.

33 Satoshi Y, Fumiya T, Kenji M, Takeshi I, Takashi Y, Hideya F. The Novel Myb Transcription Factor LCR1 Regulates the $\mathrm{CO}_{2}$-Responsive Gene Cah1, Encoding a Periplasmic Carbonic Anhydrase in Chlamydomonas reinhardtii. Plane Cell. 2004;16:1466-77.

34 Shawn D, Anthony DK, Marla JC, Robert LB. Regulation of the Cyanobacterial $\mathrm{CO}_{2}$-Concentrating Mechanism Involves Internal Sensing of NADP+ and aKetogutarate Levels by Transcription Factor CcmR. PLoS ONE. 2012;7(7):e41286

35 Chen H, Lin L, Xie M, Zhang G, Su W. De novo Sequencing, Assembly and Characterization of Antennal Transcriptome of Anomala corpulenta Motschulsky (Coleoptera: Rutelidae). PLoS ONE. 2014;10(4):e0127303.

36 Elsey D, Jameson D, Raleigh B, Cooney MJ. Fluorescent measurement of microalgal neutral lipids. J Microbiol Methods. 2007:68(3):639-42.

37 Anders S: FastQC. Babraham Bioinformatics 2015. http://www.bioinformatics. bbsrc.ac.uk/projects/fastac. Accessed 2 Jan 2015.

38 Grabherr MG, Haas BJ, Yassour M, Levin JZ, Thompson DA, Amit I, Adiconis X, Fan L, Raychowdhury R, Zeng Q, et al. Full-length transcriptome assembly from RNA-Seq data without a reference genome. Nat Biotechnol. 2011;29(7):644-52.

39 Huang Y, Niu B, Gao Y, Fu L, Li W. CD-HIT Suite: a web server for clustering and comparing biological sequences. Bioinformatics. 2010;26(5):680-2.

40 Tatusov RL, Galperin MY, Natale DA, Koonin EV. The COG database: a tool for genome-scale analysis of protein functions and evolution. Nucleic Acids Res. 2000;28(1):33-6.

41 Tatusov RL, Koonin EV, Lipman DJ. A Genomic Perspective on Protein Families. Science. 1997;278(5338):631-7.

42 Altschul SF, Madden TL, Schaffer AA, Zhang J, Zhang Z, Miller W, Lipman DJ. Gapped BLAST and PSI-BLAST: a new generation of protein database search programs. Nucleic Acids Res. 1997:25(17):3389-402.

43 Kanehisa M, Goto S, Sato Y, Furumichi M, Tanabe M. KEGG for integration and interpretation of large-scale molecular data sets. Nucleic Acids Res. 2012;40(Database issue):D109-114.

44 Moriya Y, Itoh M, Okuda S, Yoshizawa AC, Kanehisa M. KAAS: an automatic genome annotation and pathway reconstruction server. Nucleic Acids Res. 2007;35 suppl 2:W182-5.

45 Riano-Pachon DM, Ruzicic S, Dreyer I, Mueller-Roeber B. PInTFDB: an integrative plant transcription factor database. BMC Bioinformatics. 2007:8:42.

46 Li B, Dewey CN. RSEM: accurate transcript quantification from RNA-Seq data with or without a reference genome. BMC Bioinformatics. 2011;12:323.

47 Livak KJ, Schmittgen TD. Analysis of Relative Gene Expression Data Using RealTime Quantitative PCR and the 2- $\Delta \Delta C T$ Method. Methods. 2001;25(4):402-8. 